\title{
10-BIT, 1GS/s TIME-INTERLEAVED SAR ADC
}

\author{
Shravan Kumar Donthula ${ }^{1}$ and Supravat Debnath ${ }^{2}$ \\ ${ }^{1}$ Department of Electrical Engineering, \\ Indian Institute of Technology, Hyderabad, India \\ ${ }^{2}$ Integrated Sensor Systems, Centre for Interdisciplinary Programs, \\ Indian Institute of Technology, Hyderabad, India
}

\begin{abstract}
This paper describes the implementation of a 4-channel, 10-bit, $1 \mathrm{GS} / \mathrm{s}$ time-interleaved analog to digital converter (TI-ADC) in 65nm CMOS technology. Each channel consists of interleaved $\mathrm{T} / \mathrm{H}$ and $A D C$ array operating at $250 \mathrm{MS} / \mathrm{s}$, with each ADC array containing 14 timeinterleaved sub-ADCs. This configuration provides high sampling rate even though each sub$A D C$ works at a moderate sampling rate. We have selected 10-bit successive approximation $A D C(S A R A D C)$ as a sub-ADC, since this architecture is most suitable for low power and medium resolution. SAR ADC works on binary search algorithm, since it resolves 1-bit at a time. The target sampling rate was $20 \mathrm{MS} / \mathrm{s}$ in this design, however the sampling rate achieved is $15 \mathrm{MS} / \mathrm{s}$. As a result, the 10-bit SAR ADC operates at $15 \mathrm{MS} / \mathrm{s}$ with power consumption of 560 $\mu W$ at $1.2 \mathrm{~V}$ supply and achieves SNDR of $57 \mathrm{~dB}$ (i.e. ENOB 9.2 bits) near nyquist rate input. The resulting Figure of Merit (FoM) is $63.5 \mathrm{fJ} / \mathrm{step}$. The achieved DNL and INL is +0.85\-0.9 $L S B$ and $+1-1.1$ LSB respectively. The 10-bit SAR ADC occupies an active area of $300 \mu \mathrm{m} \times$ $440 \mu \mathrm{m}$. The functionality of single channel TI-SAR ADC has been verified by simulation with input signal frequency of $33.2 \mathrm{MHz}$ and clock frequency of $250 \mathrm{MHz}$. The desired SNDR of 59.3 $d B$ has been achieved with power consumption of $11.6 \mathrm{~mW}$. This results in a FoM value of 60 fJ/step.
\end{abstract}

\section{KEYWORDS}

ADC, SAR, TI-ADC, LSB, MSB, T/H, SCDAC, CDAC, SFDR, SINAD, SNR, TG, EOC, D-FF, MIM, MOM, DNL, INL.

\section{INTRODUCTION}

Time-interleaved analog to digital converters (TI-ADC) use a parallel combination of multiple sub-ADCs into a single ADC, that can improve the sampling rate compared to the sub-ADCs alone. The major advantage of time-interleaved ADC is that it can improve the sampling rate in a given technology. In wide band applications like wireless communication, serial data links require $A / D$ converters with high sampling rate to meet the channel bandwidth and power requirements. Existing high-speed $\mathrm{ADCs}$ require high power to meet the required sampling rate. However, many applications demand for high sampling rates while keeping the power consumption relatively low. In these cases, low powered sub-ADCs operating at lower sampling rates can be used with interleaving to achieve the required sampling rate. In the proposed design we have selected SAR ADC architecture for sub-ADCs, since this architecture is most suitable for low power and moderate sampling rate. The major design challenge for time-interleaved $\mathrm{ADCs}$ is matching the performance of the sub-ADCs used with respect to bandwidth, timing, offset and gain. Mismatch in the performance of sub-ADCs degrades its major specifications like SFDR, SNDR etc. required for wireless communications. The mismatch in sub-ADCs can be

David C. Wyld et al. (Eds): NLP, MLTEC, CLBD, SEAPP, NeTIoT, VLSIE, ITCS, ARIA, DMS - 2021 pp. 321-339, 2021. CS \& IT - CSCP 2021 
detected and corrected by using calibration methods either in analog or digital domain. This correction can remove the problems due to mismatch to a certain extent [6] [8]. The proposed design adds redundant sub-ADCs in each ADC array (each channel) to enable background calibration. This paper describes the design procedure for a 10-bit $1 \mathrm{GS} / \mathrm{s}$ TI-ADC and implementation of a 10-bit SAR ADC. The TI-ADCs consist of a total of $4 \times 14$ i.e. 56 timeinterleaved SAR ADCs, with each SAR ADC working at $20 \mathrm{MS} / \mathrm{s}(250 \mathrm{M} / 13 \sim 20 \mathrm{MS} / \mathrm{s})$ sampling rate to meet the overall sampling rate of $1 \mathrm{GS} / \mathrm{s}$. Each SAR ADC consists of comparator, DAC and SAR logic. The rest of the paper is organized as follows: Section-2 describes the 4-Channel TI-ADC Architecture. Section-3 describes the Implementation of 10-bit $20 \mathrm{MS} / \mathrm{s}$ SAR ADC. Section-4 provides layout design and post layout simulation results. Section5 describes the implementation of single channel TI-SAR ADC. Finally, the conclusions and future work are given in Section-5.

\section{4-ChanNel TI-ADC ARCHITECTURE}

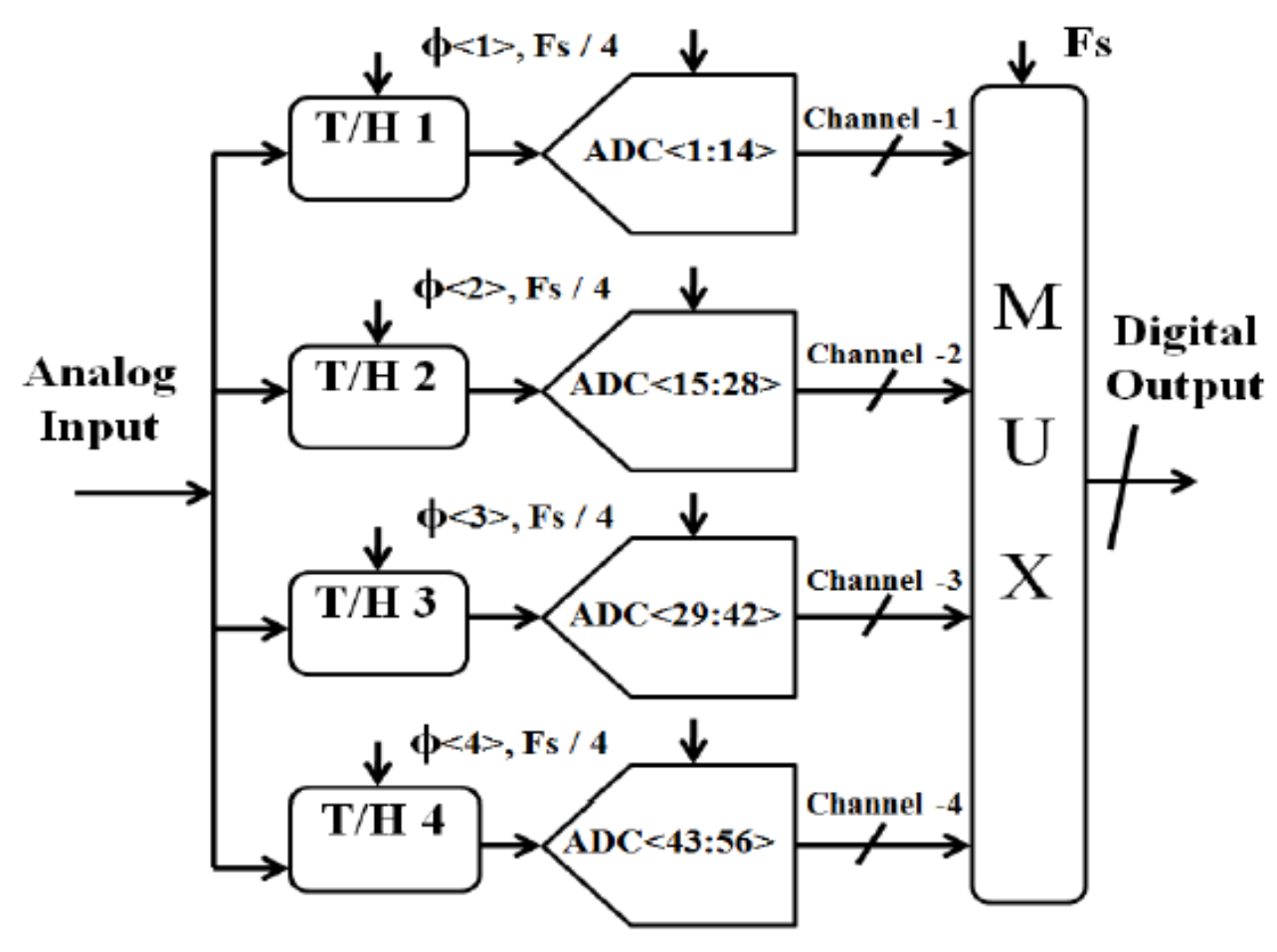

Figure 1. Architecture of 4-Channel TI-ADC.

Fig. 1 shows the architecture of the 4-channel TI-ADC, each channel consists of time-interleaved $\mathrm{T} / \mathrm{H}$ and $\mathrm{ADC}$ array, each $\mathrm{ADC}$ array uses 14 time-interleaved 10-bit SAR ADCs. The 4channels are driven by clock frequency of Fs/4, with equally spaced phases, $\phi<1>$ to $\phi<4>$. In a TI-ADC with sampling rate (Fs) of $1 \mathrm{GS} / \mathrm{s}$, each channel should operate at Fs/4 $=250 \mathrm{MS} / \mathrm{s}$ with equally spaced phases at $\phi<\mathrm{i}>=2 \pi(\mathrm{i}-1) / 4$ where $\mathrm{i}=1,2,3$ etc. $\left(0^{\circ}, 90^{\circ}, 180^{\circ}, 270^{\circ}\right)$. Sampling rate of each SAR ADC should be $20 \mathrm{MS} / \mathrm{s}$. The additional 10-bit SAR ADC used in each channel is for background calibration. Fig. 2 shows the timing diagram of 4-channel TI$\mathrm{ADC}$ at each rising edge of master clock $(\mathrm{Fs})$. The track and hold unit of each channel spends two clock cycles in track mode and two clock cycles in hold mode. This choice of timing presents a trade-off between input bandwidth and accurate sampling of the input. Since the operation of each channel is shifted with respect to the previous one by one clock cycle, at any given time, 
only two channels are in track mode. This implies that at any moment of time $\mathrm{N}=2$ ('N' represents number of channels) sampling capacitors are connected to the input. While this limits the bandwidth [5], it gives enough settling time for sampling the data (two clock cycles) to the $\mathrm{T} / \mathrm{H}$ unit. When the first $\mathrm{T} / \mathrm{H}$ goes from track mode to hold mode it has acquired a sample of the analog input over two clock periods.

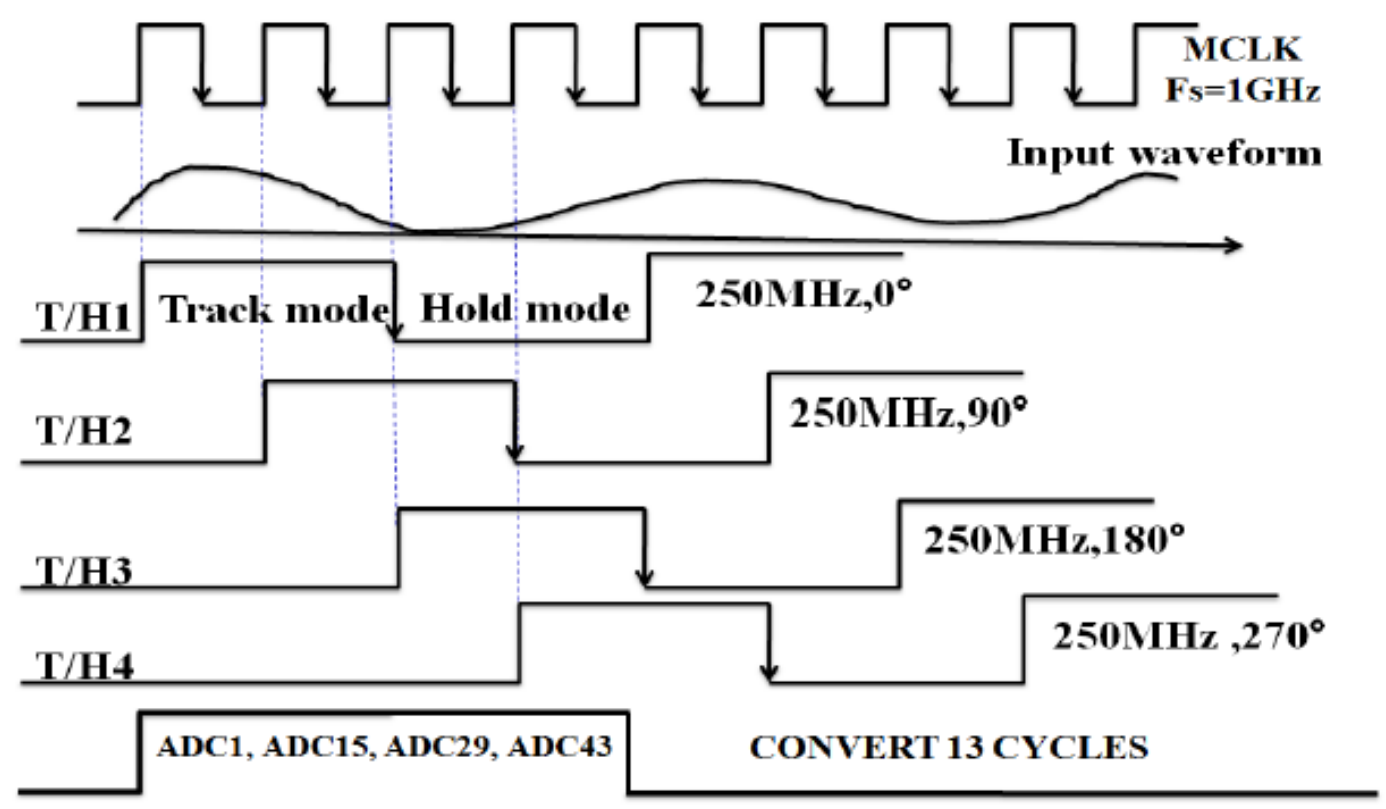

Figure 2. Timing diagram of 4-channel TI-ADC.

It is then converted to digital domain by the corresponding sub-ADCs in each channel. The master clock $(\mathrm{Fs})$ works at $1 \mathrm{GHz}$ while each track/hold and sub-ADC works at $250 \mathrm{MHz}$ clock frequency with appropriate phase shift.

\subsection{Limitations of Time-Interleaved ADC}

The optimum number of channels are chosen based on the required sampling rate. To achieve high sampling rate, increasing the number of channels is not desirable because of offset, bandwidth, timing and gain error mismatches between the channels. Mismatch in the channels degrades the performance of time-interleaved ADC. Corrections for these mismatches can be implemented to a certain extent using calibration in either digital or analog domain. However, the calibration process in time-interleaved ADCs is constrained by the number of channels.

\section{IMPLEMENTATION OF 20MS/S 10-BIT SAR ADC}

A Successive approximation register (SAR) ADC works on binary search algorithm principle. Fig. 3 shows the block diagram of fully differential 10-bit SAR ADC. It consists of comparator, capacitive DAC (CDAC) \& SAR logic. Binary search algorithm proceeds along the following steps to convert the analog input into its equivalent digital output [13]. The first step is to sample the differential inputs during sampling phase using CDAC. Next step is to set the 10-bit register in SAR logic to mid code (i.e., 1000...00, here MSB is ' 1 '), this forces the 10-bit CDAC to $\mathrm{V}_{\text {pref }}$ 12 where $\mathrm{V}_{\text {pref }}$ is positive reference voltage to the CDAC. The comparator compares the 
differential DAC outputs (i.e., $V_{p}=-V_{\text {inp }}+V_{\text {pref }} / 2 \& V_{n}=-V_{\text {inn }}+V_{\text {pref }} / 2$ ). If the $V_{n}>V_{p}$ (i.e., $V_{\text {inp }}$ $>V_{\text {inn }}$ ) then MSB will remain at active high otherwise it will be forced to active low. After resolving MSB bit, the SAR logic moves to next bit, it forces that bit active high and does another comparison, this process is repeated for 10-bits till it reaches to least significant bit (LSB). Then 10-bit digital output code is available at end of conversion (EOC).

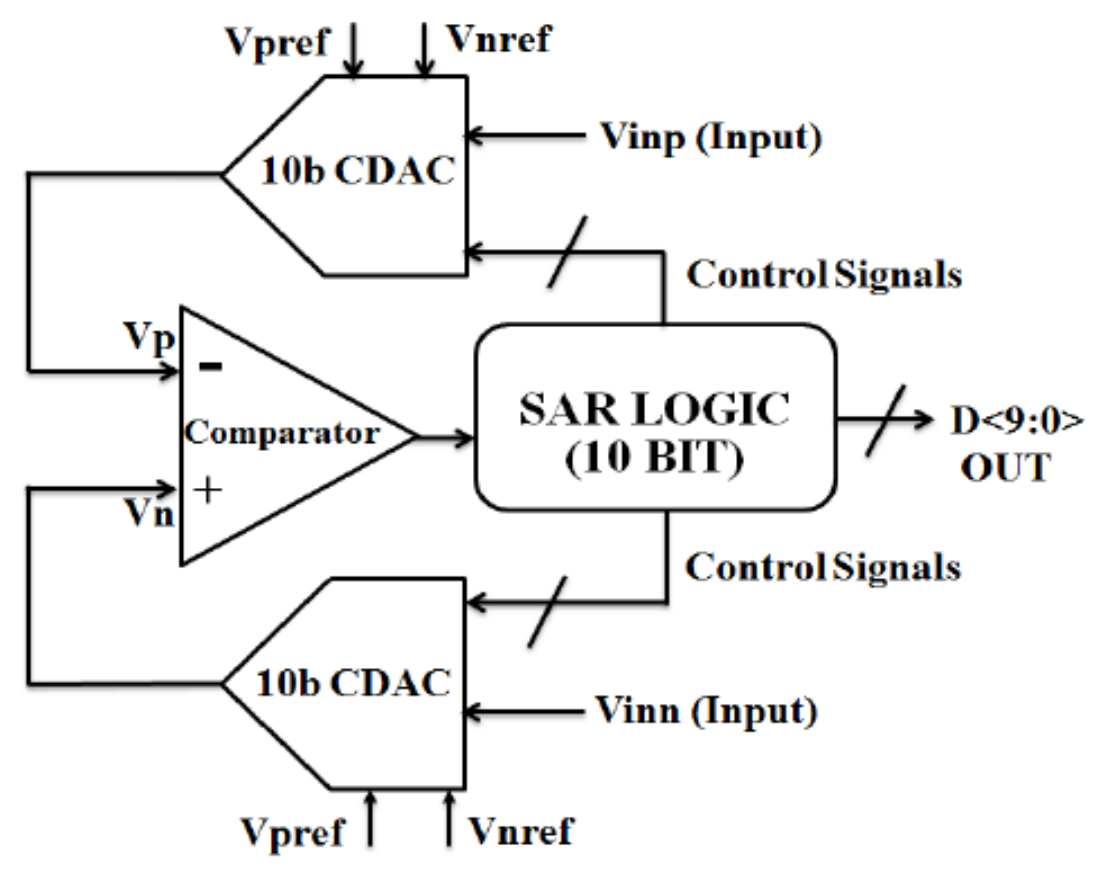

Figure 3. Block diagram of 10-bit SAR ADC

Since, SAR ADC resolve the input one bit at a time, the complexity and power consumption is lower at expense of reduced sampling rate. 10-bit SAR ADC requires the $10+3$ clock cycles to get the digital output for given analog input inclusive of two cycles for sampling the input with comparator offset calibration before the conversion takes place and another cycle for data latch after the conversion. This cycle signal is called end of conversion (EOC). 


\subsection{0-bit Fully Differential Capacitive DAC}

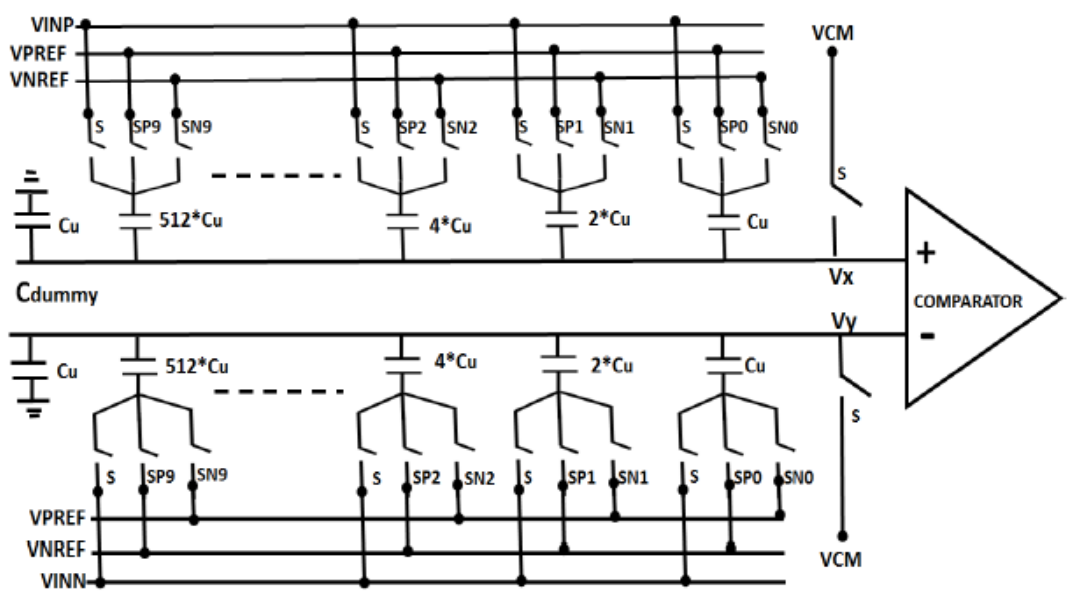

Figure 4. Architecture 10-bit fully differential CDAC

Fig. 4 shows architecture of 10-bit fully differential capacitive DAC (CDAC). It performs both sampling $(\mathrm{S} / \mathrm{H})$ operation as well as DAC operation. CDAC switching mechanism is controlled with SAR logic based on the comparator decision. In 10-bit CDAC, the capacitor network is in binary weighted fashion with dummy capacitor, Where $\mathrm{C}_{\mathrm{u}}$ is a unit capacitor. For simplicity, the explanation given here is for a single sided CDAC. The same is applicable for a fully differential CDAC. During the sampling phase (S), the top plates of the capacitors in network are connected to common mode voltage $\left(\mathrm{V}_{\mathrm{cm}}=0.6 \mathrm{~V}\right)$ through a switch and bottom plates are charged to the input voltage $\left(\mathrm{V}_{\mathrm{inp}}\right)$. The total charge on the capacitor during sampling phase is calculated by $\mathrm{Q}_{\text {tot }}$ $=2^{\mathrm{n}} * \mathrm{C}_{\mathrm{u}}\left(\mathrm{V}_{\mathrm{cm}}-\mathrm{V}_{\mathrm{inp}}\right)$ for an n-bit CDAC. During the next cycle, the top plate switch is opened before the SAR logic sets the MSB bit to high (' 1 ') thus the bottom plate of the MSB capacitor is now connected to $\mathrm{V}_{\text {pref }}(1.2 \mathrm{~V})$. The remaining capacitors are connected to $\mathrm{V}_{\text {nref }}(0 \mathrm{~V})$. Since the top plate is connected to a high input impedance of the comparator, the total charge on capacitors remains the same [14]. However, switching causes the charge to redistribute resulting in the top plate potential going to $\mathrm{V}_{\mathrm{x}}$. The total charge on top plate capacitor is given by

$$
\begin{aligned}
& \mathrm{Q}_{\text {tot }}=\mathrm{Q}_{\mathrm{MSB}}+\ldots . .+\mathrm{Q}_{\mathrm{LSB}}+\mathrm{Q}_{\text {dummy }} \\
& 2^{\mathrm{n}} \mathrm{C}_{\mathrm{u}}\left(\mathrm{V}_{\mathrm{cm}}-\mathrm{V}_{\text {inp }}\right)=2^{\mathrm{n}-1} \mathrm{C}_{\mathrm{u}}\left(\mathrm{V}_{\mathrm{x}}-\mathrm{V}_{\text {pref }}\right)+2^{\mathrm{n}-1} \mathrm{C}_{\mathrm{u}}\left(\mathrm{V}_{\mathrm{x}}\right) \\
& \mathrm{V}_{\mathrm{x}}=-\mathrm{V}_{\text {inp }}+\mathrm{V}_{\mathrm{cm}}+\mathrm{V}_{\text {pref }} / 2
\end{aligned}
$$

If $\mathrm{V}_{\mathrm{x}}<\mathrm{V}_{\mathrm{cm}}$, it means $\mathrm{V}_{\text {inp }}>\mathrm{V}_{\text {pref }} / 2$ and the most significant bit (MSB) value is set to high, otherwise it is reset to low (' 0 '). In next cycle the second MSB bit is set to high, which results in a comparison with either $3 \mathrm{~V}_{\text {pref }} / 4$ or $\mathrm{V}_{\text {pref }} / 4$, depending on the value of MSB bit, this process continues till the CDAC converges to $\mathrm{V}_{\mathrm{cm}}$ (common mode voltage) and the LSB is determined. The major advantage of capacitive DAC is that it is less sensitive to parasitic effects across the capacitor array, which improves linearity (monotonic) at the DAC outputs.

\subsection{Selection of Unit Capacitor $\left(C_{u}\right)$ and Switch}

The value and type of unit capacitor is chosen based on area and mismatch data given in UMC65nm CMOS technology. This technology has two different types of capacitors (i) MOM 
Capacitor (ii) MIM Capacitor. MIM capacitor is more accurate compared to MOM capacitor, but it occupies more area. The value of unit capacitor is limited by thermal and quantization noise (i.e., the sum of rms thermal noise and rms quantization noise to be less than $\mathrm{LSB} / 2$ ). $\mathrm{KT} / \mathrm{C}_{\text {total }}+$ $\mathrm{LSB}^{2} / 12<(\mathrm{LSB} / 2)^{2}$, where $\mathrm{LSB}=1.2 / 2^{10}=1.17 \mathrm{mV}$, therefore $\mathrm{C}_{\text {total }}$ should be greater than 100 $\mathrm{fF}$. Also, the total capacitance for 10 -bit CDAC is $2^{10} * \mathrm{C}_{\mathrm{u}}=\mathrm{C}_{\text {total }}$, where $\mathrm{C}_{\mathrm{u}}$ unit capacitance. This gives a unit capacitance of $\mathrm{C}_{\mathrm{u}}>100 \mathrm{aF}$ [15]. Choosing a lower value capacitance reduces the switching power and area but mismatch between capacitor arrays become very high which leads to non-linearity. In this design considering the area constraint, switching power and mismatch factors, the chosen unit capacitance $\left(\mathrm{C}_{\mathrm{u}}\right)$ value is $10 \mathrm{fF}$ which occupies an area of $4 \mu \mathrm{m}$ $* 4 \mu \mathrm{m}$. Transmission gates (TG) were used as switches to meet the settling error within LSB/2, given the settling time $(800 \mathrm{pS})$ during the sampling phase. Considering the unit capacitance of $10 \mathrm{fF}$, the total capacitance during the sampling phase, $\mathrm{C}_{\text {hold }}=2^{10} * 10 \mathrm{fF}=10.24 \mathrm{pF}$. The settling time of DAC output is $4 \mathrm{~ns}\left(5^{*} \mathrm{R}_{\text {on }} * \mathrm{C}_{\text {hold }}=4 \mathrm{~ns}\right)$ with clock frequency of $\left.250 \mathrm{MHz}\right)$ so, the ONresistance of switch should be less than $78 \Omega$ (i.e., Ron $<78 \Omega$ ). Which leads to TG transistor (Both NMOS and PMOS) sized with $\mathrm{W}=10 \mu \mathrm{m}$ and $\mathrm{L}=0.1 \mu \mathrm{m}$.

\subsection{Selection of Comparator Architecture}

The comparator is a key component in an analog to digital converter (ADC). Every ADC contains at least one comparator (1-bit A/D converter). It must be able to resolve very small analog voltage and convert it into a rail-to-rail digital output. This small input voltage is known as the resolution of the comparator. The basic function of the comparator is to compare the differential input signal with the reference threshold and to give a digital decision accordingly. The architecture of the comparator is chosen based on resolution, speed, offset voltage and power dissipation. A dynamic latch comparator is most widely used for high speed and low power consumption. The main disadvantage of latch type comparators is their high input offset voltage due to mismatch in transistors and also the kick back noise effect at the inputs. To reduce the input offset voltage and the effect of kick back noise, a preamplifier is used in front of the dynamic latch. The requirements from the comparator are:

- Resolution $(\mathrm{LSB})=1.17 \mathrm{mV}$

- Input offset voltage and input integrated noise $<\mathrm{LSB} / 2$

- Settling time $<800 \mathrm{ps}$, considering $250 \mathrm{MHz}$ as clock frequency

\subsection{Architecture of Preamplifier Based Dynamic Latch Comparator}

Fig. 5 shows the schematic of preamplifier based dynamic latch comparator. It consists of three stages viz preamplifier, dynamic latch and SR latch. Preamplifier is a differential amplifier with resistive load $(\mathrm{R} 0, \mathrm{R} 1=5 \mathrm{~K} \Omega)$ with wide bandwidth and relatively small gain to achieve high speed as shown in Fig. 5a. The dynamic latch consists of two cross-coupled CMOS inverters used for regeneration as shown in Fig. 5b. It operates in either one of the following two modes:

- $\quad$ Rest phase $(\mathrm{CLK}=\mathrm{LOW})$

- Evaluation phase $(\mathrm{CLK}=\mathrm{HIGH})$ 


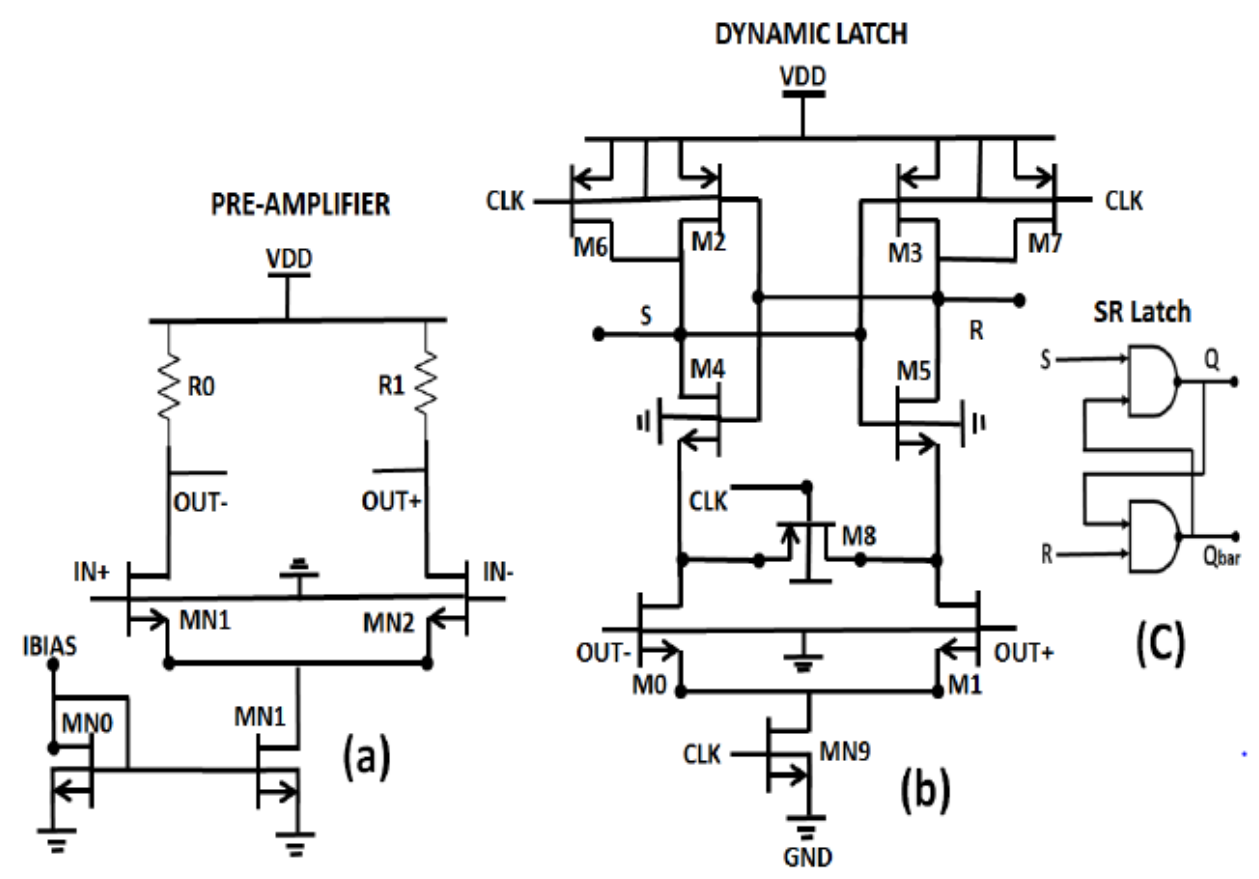

Figure 5. Schematic of preamplifier based dynamic latch

During the reset phase, the output nodes of cross coupled inverters (M2-M5) are reset to $\mathrm{V}_{\mathrm{DD}}$ through the reset transistors M6 and M7. During the evaluation phase, the tail transistor MN9 is turned on. The input transistor pairs (M0, M1) start discharging at different time rates depending on the applied input voltage. This initiates positive feedback, enhancing the small differential voltage to a full swing differential output. The mismatch of transistor size in differential pair (M0, M1) and threshold voltage variation between M4, M5 transistor can lead to high input offset voltage. This offset voltage can be reduced by keeping a preamplifier in front of the dynamic latch which also prevents kick back noise to the differential inputs. The gain of the preamplifier is $19.5 \mathrm{~dB}$ with unity gain bandwidth (UGB) of $5 \mathrm{GHz}$, input offset voltage of $\pm 872 \mu \mathrm{V}$ and input integrated noise $(100 \mathrm{~Hz}$ to $5 \mathrm{GHz})$ of $715 \mu \mathrm{V}$ (rms). 


\subsection{0-bit Successive Approximation Register Logic}

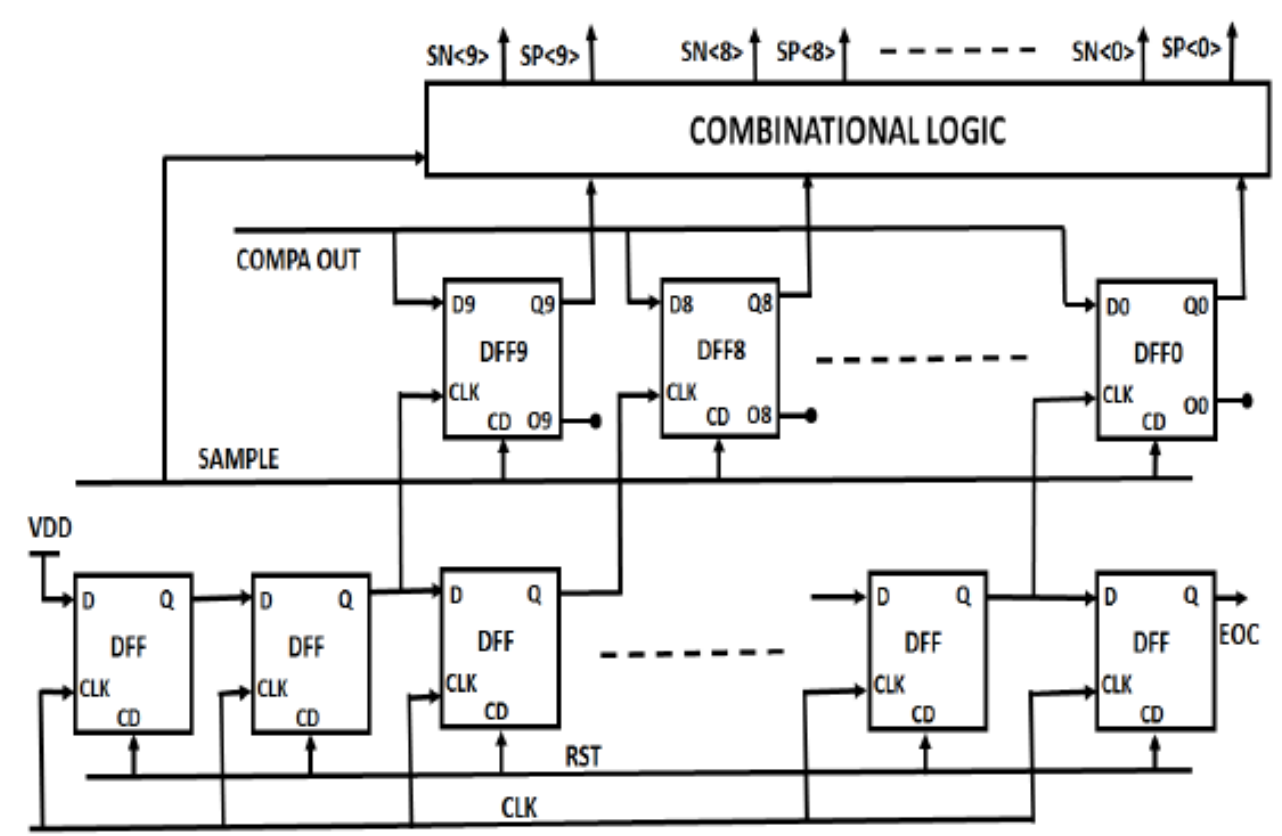

Figure 6. Block diagram of 10-bit SAR logic

There are two kinds of approaches to design SAR logic. One is a method proposed by Anderson [17] and the other is Rossi and Fucili method [18]. Fig. 6 shows 10-bit SAR logic proposed by Anderson. It is based on shift registers and combination logic. In this design, the binary search 10-bit SAR logic is implemented using this approach. The proposed 10-bit SAR logic requires at least $2 * 10=20 \mathrm{D}-\mathrm{FF}$. One chain of $10 \mathrm{D}$-FFs for storing the conversion results and another chain of $10 \mathrm{D}-\mathrm{FFs}$ for performing the shift operation and generation of necessary control signals $(\mathrm{SN}<$ 9:0 >, SP $<$ 9:0 >) for DAC operation using combinational logic. SAR logic performs three main operations, (i) It shifts the initial mid code (i.e.,1000...00, here MSB is ' 1 ') to the right by one bit (ii) It loads the result from the comparator (COMPA OUT) during the positive edge triggering of next nearest bit (iii) Finally it holds the converted bits. After 10 clocks, shift registers generate pulse called end of conversion (EOC), which means that the whole conversion is completed. The EOC also indicate the start of the next sampling.

\section{Layout Design \& Post Layout Simulation Results}

\subsection{Transient response of 10-bit CDAC}

Fig. 7 shows the transient response of differential CDAC $\left(V_{x}, V_{y}\right.$ nodes shown in Fig. 4) with DC input voltages $\mathrm{V}_{\text {inp }}=1.1 \mathrm{~V}$ and $\mathrm{V}_{\mathrm{inn}}=0.1 \mathrm{~V}$ at $10 \mathrm{MS} / \mathrm{s}$ sampling rate. The corner (typ, min, and max) and monte-carlo simulations have been carried out for differential CDAC and the 


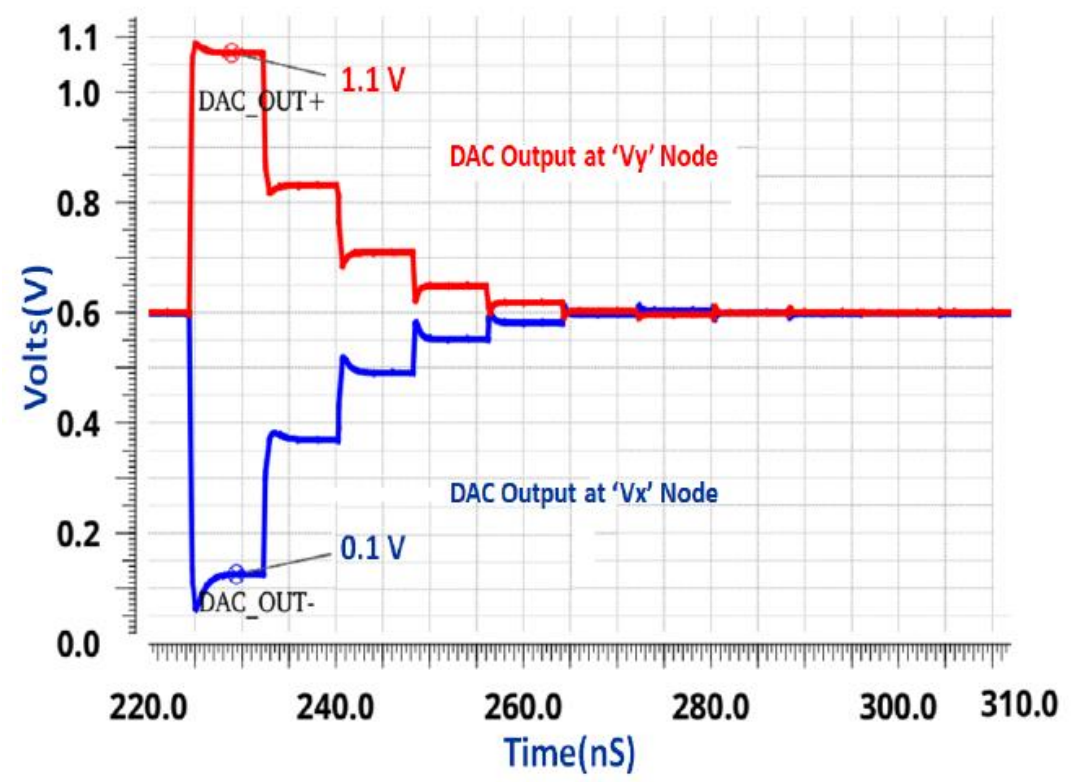

Figure 7. Transient response of 10-bit CDAC

output voltage variation of about $\pm 900 \mu \mathrm{V}$ at maximum input voltage (i.e., <LSB) is seen. DAC output voltages are settled within $0.5 \mathrm{LSB}$ of the final value. The dynamic power (i.e., $\mathrm{C}_{\text {total }}$ * $\mathrm{V}_{\mathrm{DD}}^{2} * \mathrm{Fs}$ ) of 10 -bit CDAC is $150 \mu \mathrm{W}$ with $1.2 \mathrm{~V}$ supply at $10 \mathrm{MS} / \mathrm{s}$.

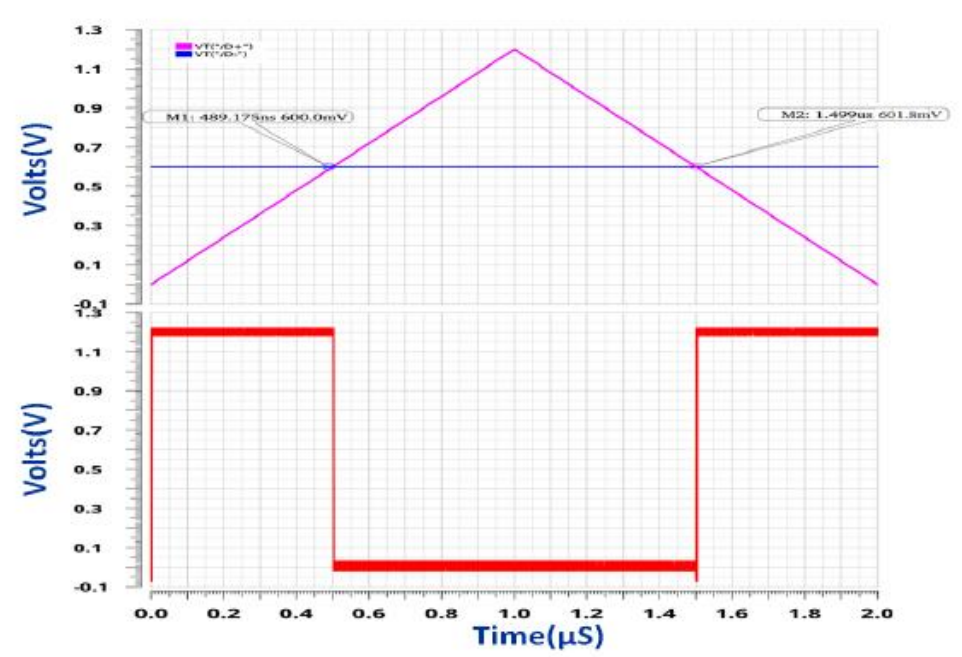

Figure 8. Transient response of dynamic comparator

Fig. 8 shows the transient response of comparator with triangular input signal and with a common mode voltage of $0.6 \mathrm{~V}$. To estimate the input offset voltage of preamplifier-based latch comparator, Monte-Carlo simulations were carried out (number of runs $=50$ ) and the comparator output transition were seen to be shifted towards positive and negative side in time which results an input offset voltage deviation of $\pm 872 \mu \mathrm{V}$ about the mean value respectively as shown in Fig. 9 


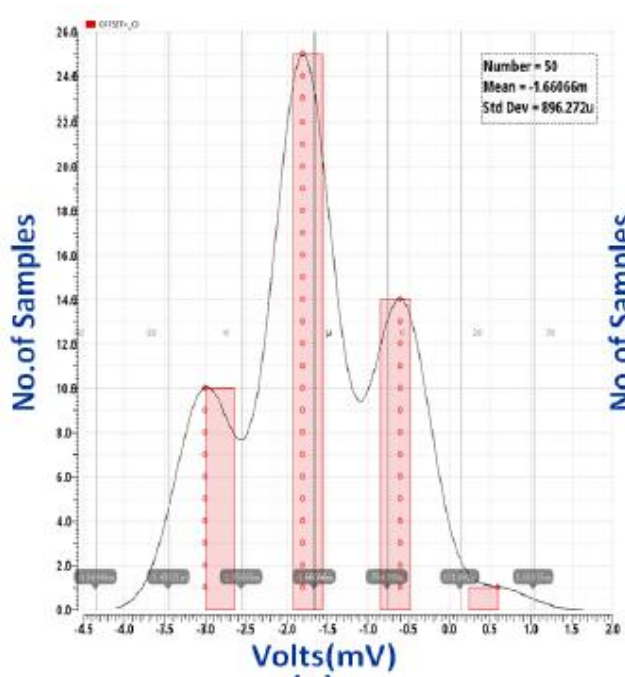

(a)

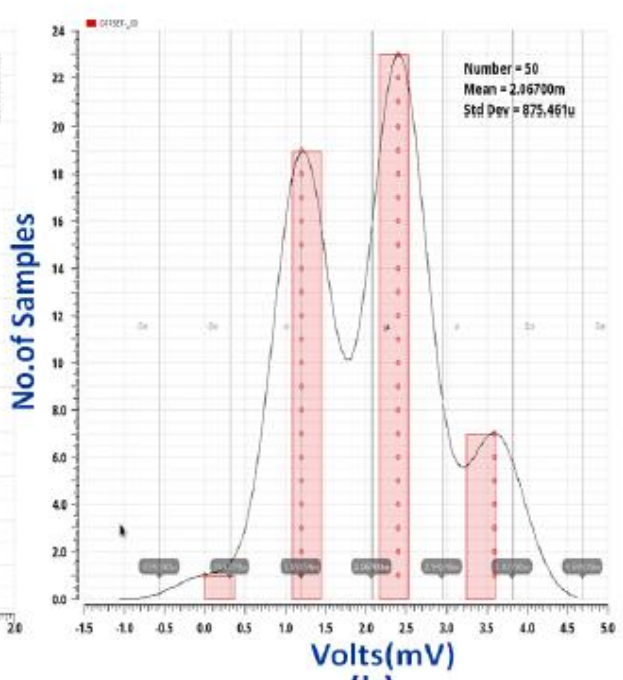

(b)

Figure 9. Preamplifier based dynamic latch comparator input offset voltage (a) at negative terminal (b) at positive terminal

\subsection{Transient Response of 10-bit SAR Logic}

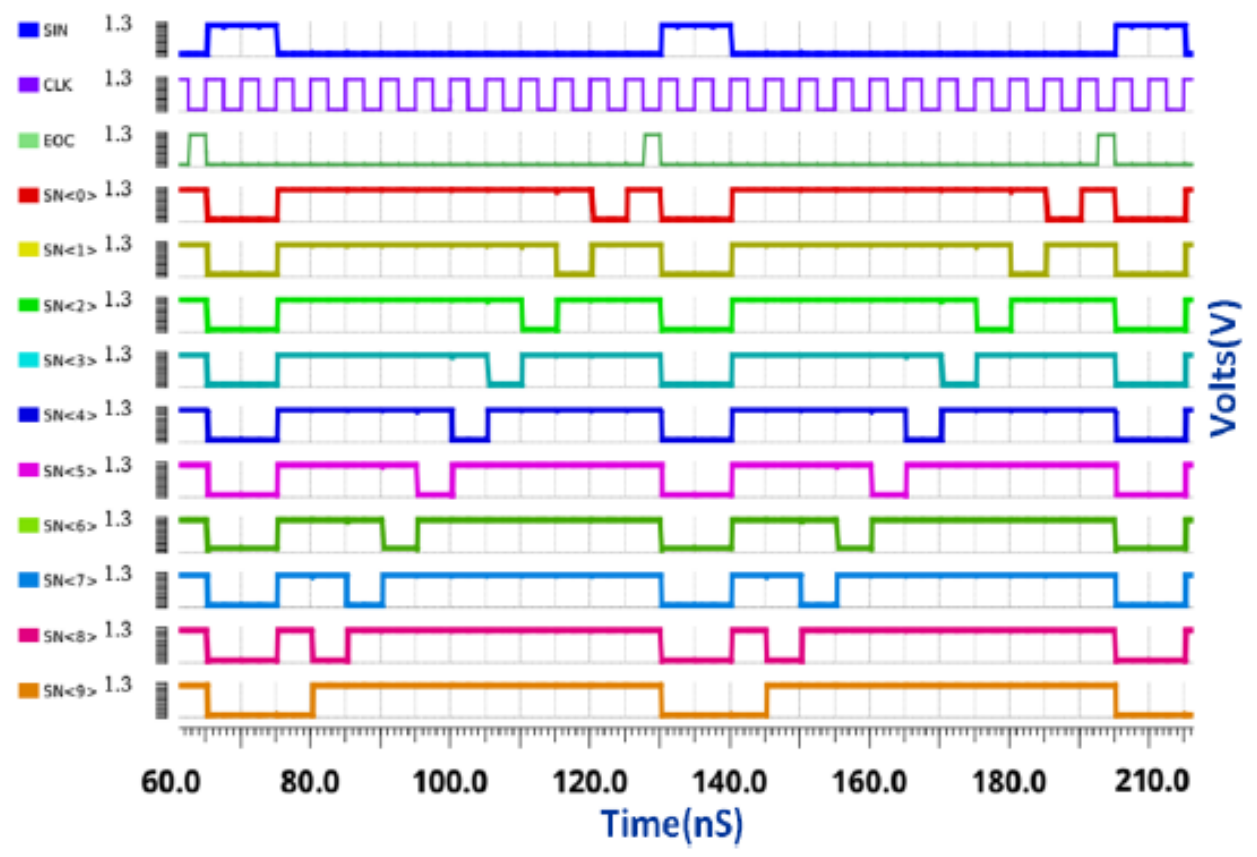

Figure 10. Transient response of 10-bit SAR logic

Fig. 10 shows the transient response of 10-bit SAR logic. The clock frequency of $250 \mathrm{MHz}$ pulse input (period $=4 \mathrm{~ns}$ ) is given to MOD-13 counter to generate the sample pulse (SIN). It repeats for every 13 cycles (i.e $13 * 4 \mathrm{~ns}=52 \mathrm{~ns}$ ). 10-bit shift register used for generating the EOC pulse $\left(\mathrm{T}_{\text {pulse }}=4 \mathrm{~ns}\right)$ just before the next sampling. The shift register and combination logic is used for generating control signals $(\mathrm{SN}<0>, \mathrm{SN}<1>. . \mathrm{SN}<9>$ and $\mathrm{SP}<0>, \mathrm{SP}<1>. . \mathrm{SP}<9>)$ based on comparator decision (COMPA OUT). 


\subsection{Layout Design \& Dynamic Performance of 10-bit SAR ADC}

Fig. 11 shows the layout of 10-bit SAR ADC. In this design, the METAL stack with maximum metal width is used for routing critical nets like differential CDAC outputs, comparator output in a symmetric manner to avoid the output voltage mismatch, delay due to parasitic effects. All control signals which control the switching of CDAC are laid out symmetrically to meet the equal delay effect. The total area occupied by 10-bit SAR ADC is $300 \mu \mathrm{m} * 440 \mu \mathrm{m}$.

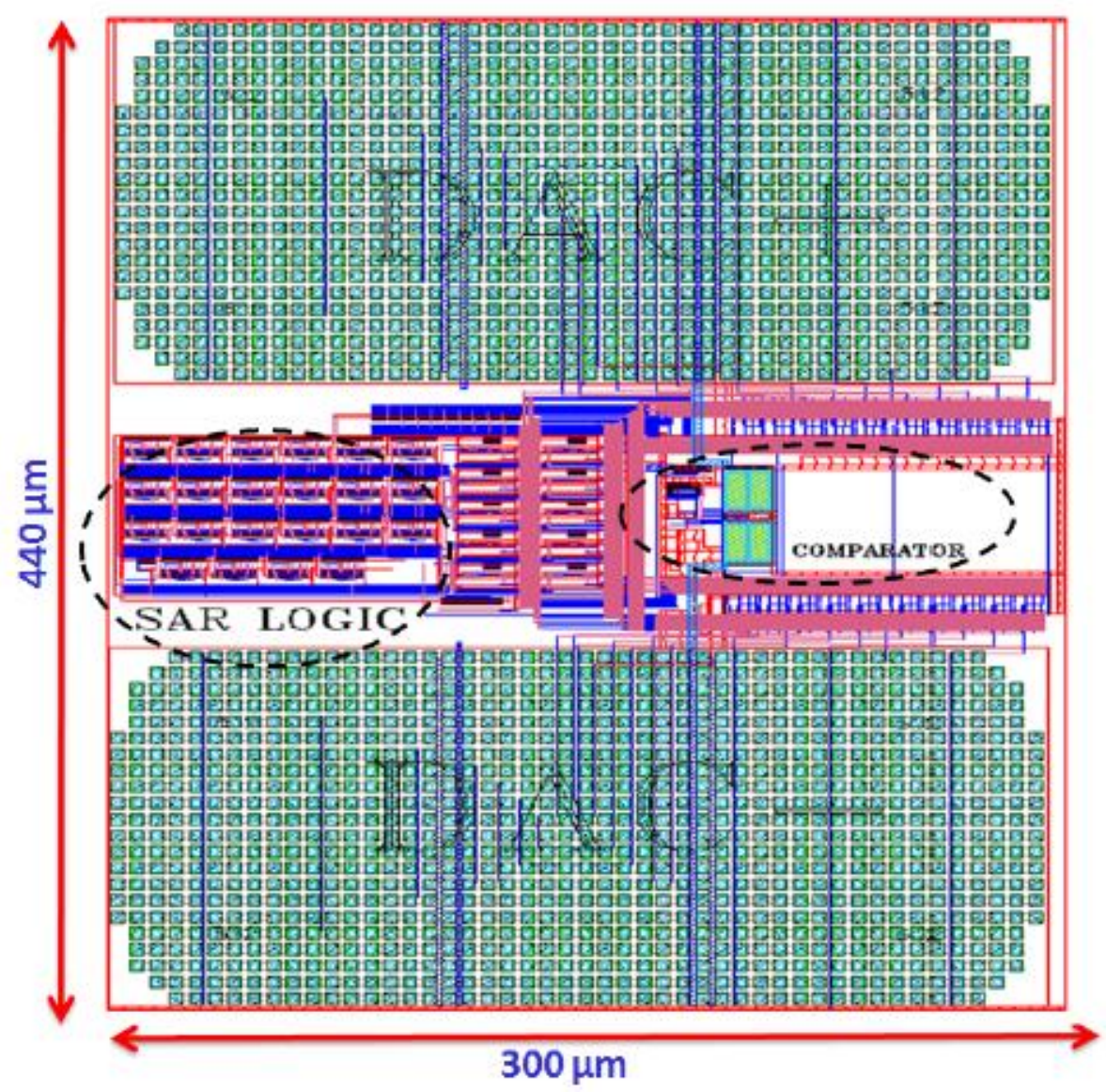

Figure 11. Layout of 10-bit SAR ADC

The coherent sampling method is used for evaluating dynamic performance of ADC as shown in Fig. 12b, the transient noise simulation is carried out to include quantization, thermal noise effects $\left(F_{\max }\right.$ is chosen as maximum clock frequency given for ADC i.e., $\left.250 \mathrm{MHz}\right)$. The following inputs are given for evaluating the dynamic performance of 10-bit SAR ADC. $V_{\text {in }}=1$ $\mathrm{V}_{\mathrm{p}-\mathrm{p}}, \mathrm{F}_{\text {in }}=\mathrm{F}_{\mathrm{s}} / 2$ (i.e., nyquist rate input), $\mathrm{F}_{\mathrm{s}}=15 \mathrm{MS} / \mathrm{s}$ and no. of points is 128 . The achieved SFDR is $64.5 \mathrm{~dB}$ and $\mathrm{SNDR}$ of $57 \mathrm{~dB}(\mathrm{ENOB}=9.2$ bits). 


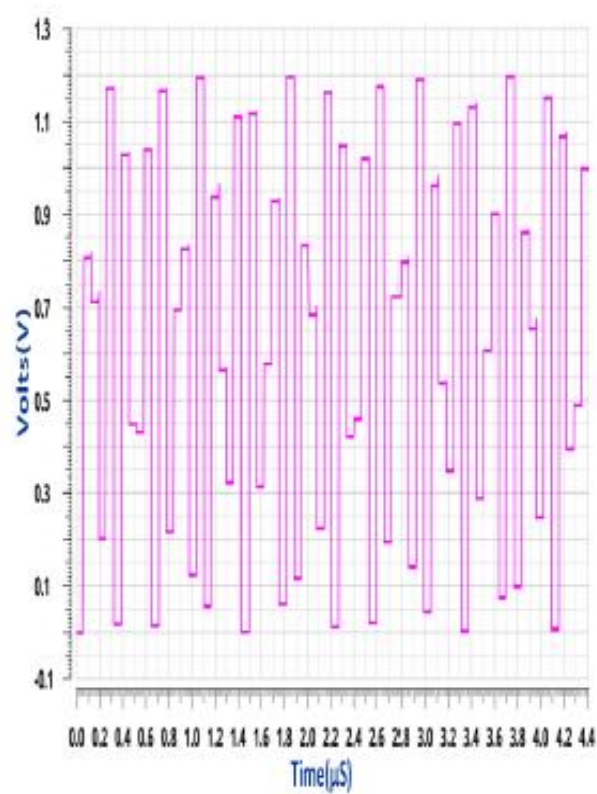

(a)

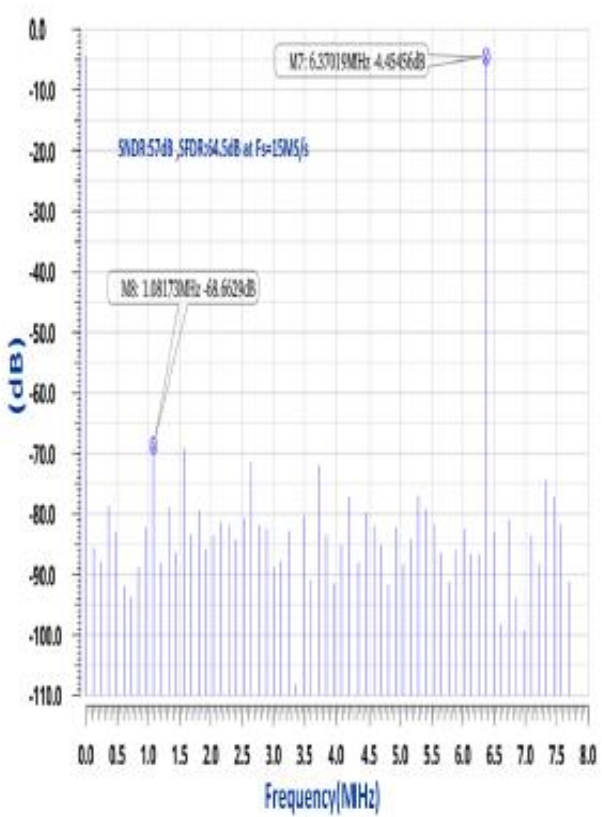

(b)

Figure 12. 10-bit SAR ADC (a) Transient response (b) Output Spectrum

\subsection{Static Performance of 10-bit SAR ADC}

The evaluation of static performance (DNL and INL) of 10-bit SAR ADC is done by using endpoint method with ramp input. The following inputs are given for evaluating the static performance of 10-bit SAR ADC, ADC sample rate is $\mathrm{F}_{\mathrm{s}}=15 \mathrm{MS} / \mathrm{s}$ or $\mathrm{T}_{\mathrm{s}}=66 \mathrm{~ns}$, resolution of $\mathrm{ADC}$ is $1.17 \mathrm{~m} \mathrm{~V}$, for LSB/4 measurement the resolution per code becomes 4 samples/code and ramp duration per code will be $264 \mathrm{~ns}$ (i.e., $4 * 66 \mathrm{~ns}$ ). So, ramp slope is $1.17 \mathrm{mV} / 0.264 \mu \mathrm{s}$. The achieved DNL of $+0.85 \backslash-0.9$ LSB is shown in Fig. 13a and INL of $+1.061 \backslash-1.124$ LSB is shown in Fig. 13b. The major INL and DNL jump occurs at first and second MSB transition, which are due to capacitor mismatches [12]. 


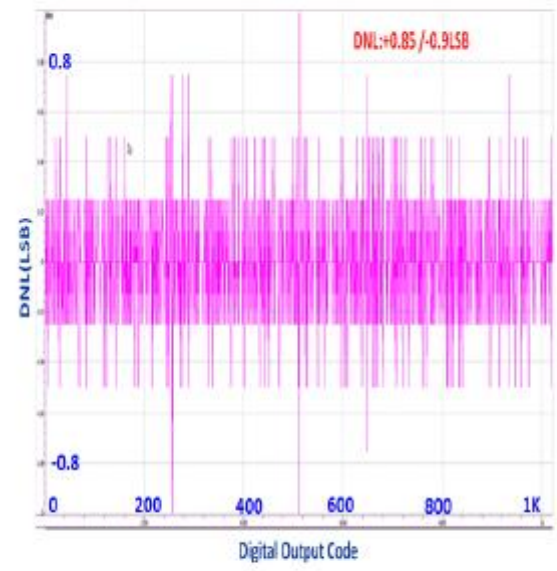

(a)

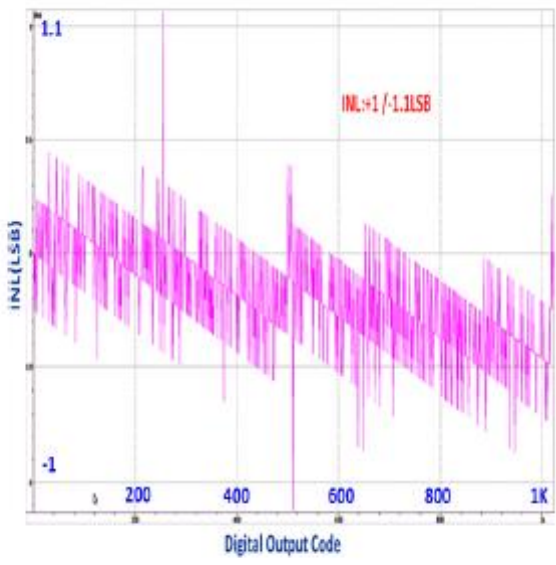

(b)

Figure 13: Static performance of 10-bit SAR ADC (a) DNL (b) INL

\section{Architecture of Single Channel TI-SAR ADC}

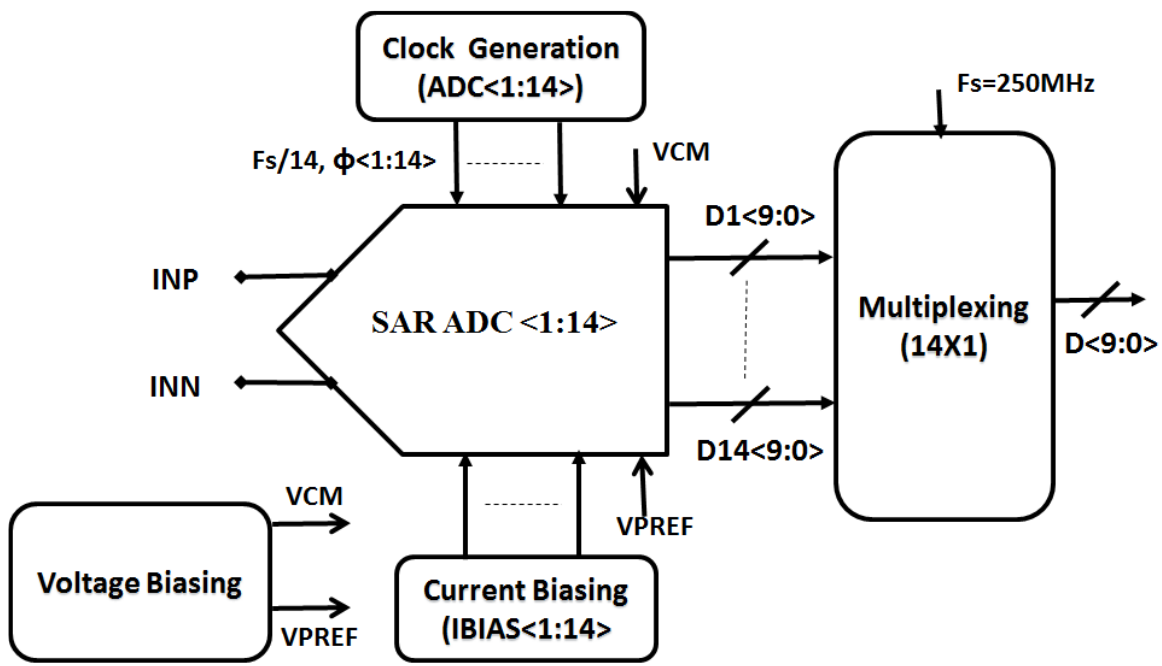

Figure 14. Block diagram of single channel 10 bit, TI-SAR

A Single Channel TI-SAR ADC architecture consist of the following sub-modules as shown in Fig. 14.

- Current Biasing

- Clock generation / Timing scheme

- Digital Multiplexing

\subsection{Current Biasing}

Fig. 15 shows the current basing circuit of single channel TI-SAR ADC. The two-stage differential amplifier (error amplifier) with gain of $65 \mathrm{~dB}$ is used to create the voltage controlled current source (voltage to current converter). In Fig. 15 shows the voltage to be converted is 
applied to noninverting terminal of the amplifier. The inverting terminal of the amplifier is connected in negative feedback viz resistor and transistor MN0. The output of amplifier drives the input gate of the transistor MNO. The error amplifier will force the required gate voltage such that the voltage across resistor R0 need to be $V_{\text {ref }}$ is equal to $0.6 \mathrm{~V}$, which results the reference current in R0 and MN0 will be $120 \mu \mathrm{V}$ (i.e., $\mathrm{V}_{\text {ref }} / \mathrm{R} 0$ ). Further this reference current is pass through PMOS current mirror (M0 to M14) and mirror current is scaled down to $50 \mu \mathrm{A}$ by sizing the PMOS transistor, finally the bias currents are (IBIAS $<1>$ to IBIAS $<14>$ ) fed to comparators of 14 TI-SAR ADC.

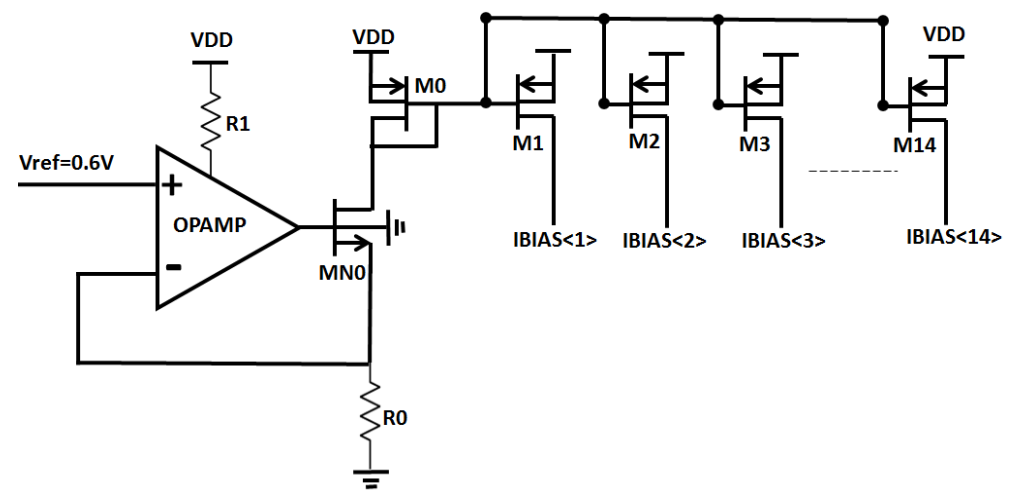

Figure 15. Schematic of voltage to current converter

\subsection{Timing Scheme/Clock Generation}

Fig. 16 shows the timing scheme of single channel time-interleaved SAR ADC. It is based on shift register. In this design, the timing signals (ADC $<1>$ to ADC $<14>$ ) for operation of TISAR ADC are generated by cascading the DFFs, so that the output from the previous flip-flop becomes input to the next flip-flop. While performing shift operation the timing signals are shifted by one clock period, where the clock (CLK) operates at $250 \mathrm{MHz}$ (i.e., period of $4 \mathrm{~ns}$ ) and the reference sample pulse is generated by MOD-14 counter, which repeats for every 14 clock cycles.

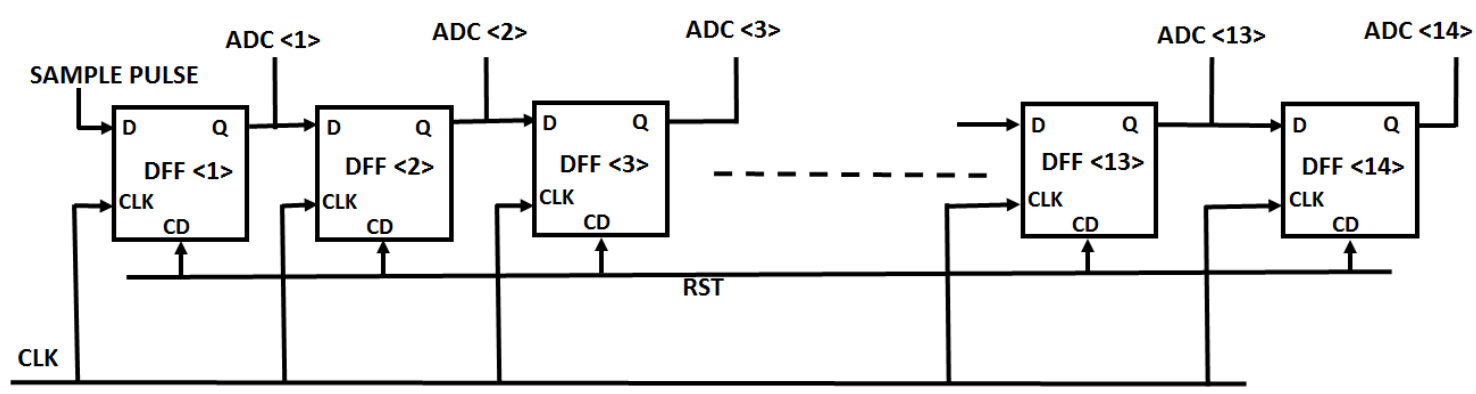

Figure 16. Block diagram of timing scheme

Fig. 17 shows the transient response of timing scheme for 14 time-interleaved SAR ADC, here the CLK operates at $250 \mathrm{MHz}$ and reference sample pulse (SAMPLE PULSE) which repeats for every 14 clock cycles. The timing signals for operation of TI-SAR ADC (ADC $<1>$ to ADC $<14$ $>$ ) are shifted by one clock period without overlapping. 


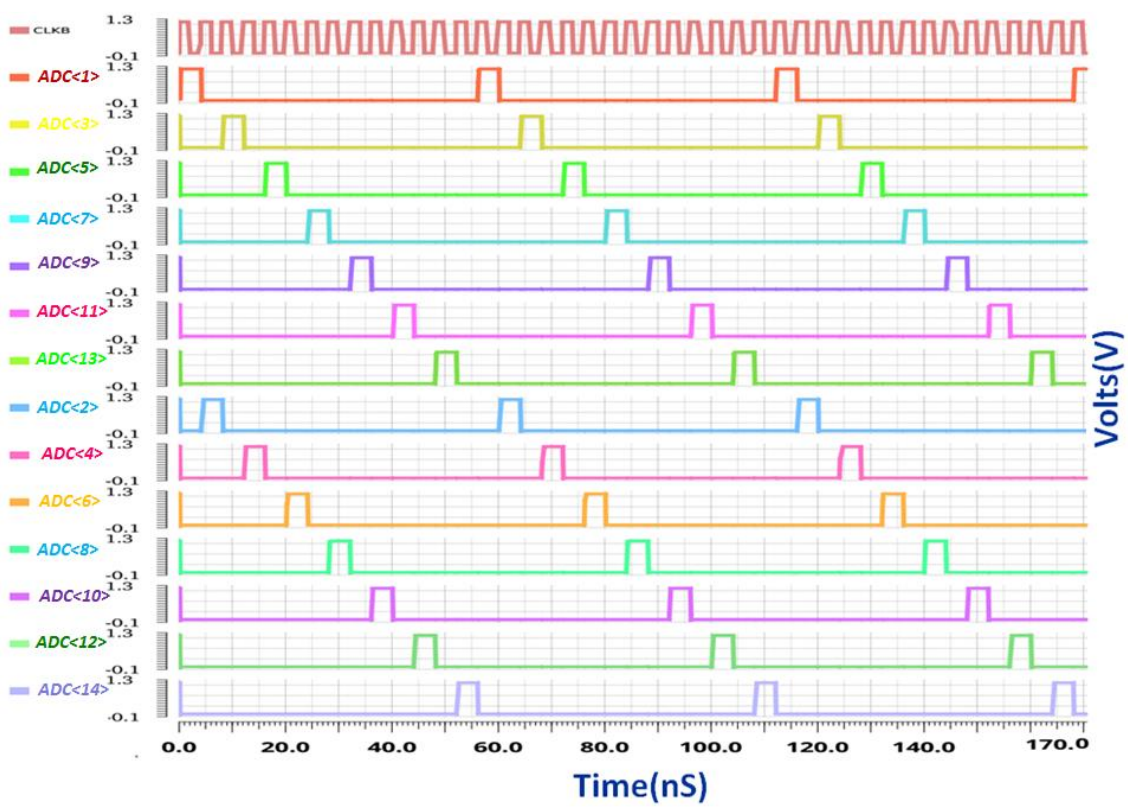

Figure 17. Transient response of timing scheme

\subsection{Digital Multiplexing}

Fig. 18 shows the block diagram of $14 \times 1$ digital multiplexing. In this design, the output data from each 10-bit SAR ADC (D $<1: 10>$ ) is available at EOC. During rising edge of EOC the digital data from 14 TI-SAR ADC is latched into corresponding D-FFs (DFF1< $1: 10>$ to DFF14 $<1$ : $10>$ ). The latched data (D1<1: $10>$ to D14< $1: 10>$ ) is fed to corresponding $14 \times 1$ multiplexer and finally the multiplexed output data read in parallel combination, which is controlled by an MOD-14 counter $\left(\mathrm{S}<1: 4>\right.$ ) operates at $\mathrm{F}_{\mathrm{s}}=250 \mathrm{MHz}$.

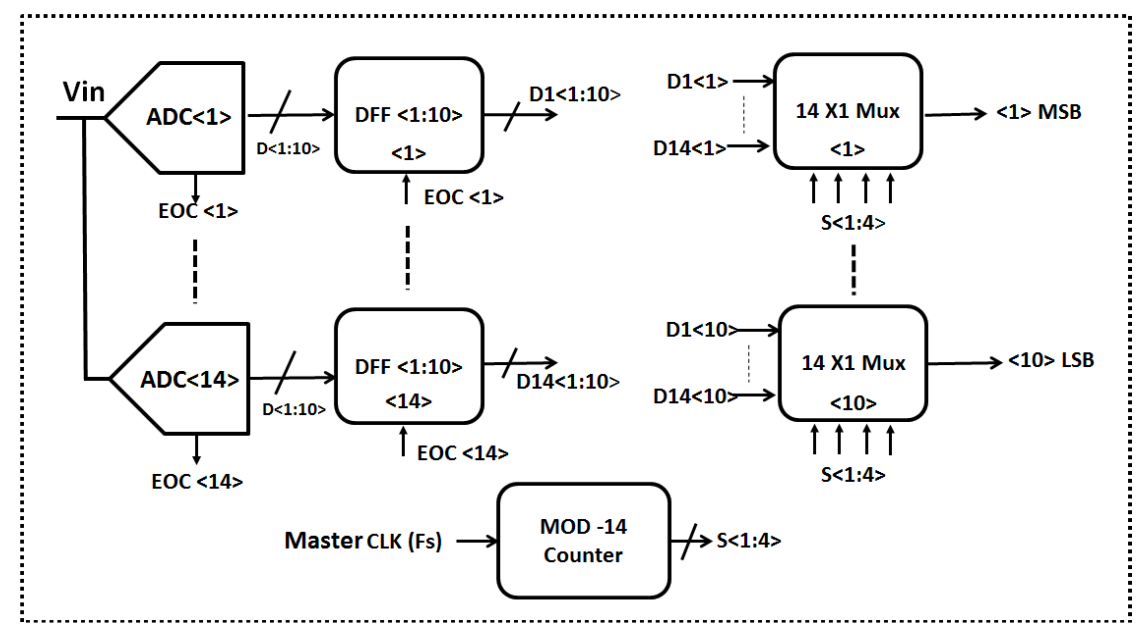

Figure 18. Block diagram of $14 \times 1$ digital multiplexing 


\section{Simulation Results of 14 TI-SAR ADC}

\subsection{Dynamic performance}

The ideal 10-bit DAC is used to reconstruct sampled analog output voltages from the 10-bit digital outputs $(\mathrm{D}<9: 0>)$ as shown in Fig. 19a, here $\mathrm{D}<9>$ represents the sign bit. The coherent sampling method is used for evaluating dynamic performance of TI-SAR ADC as shown in Fig. 19b. The transient noise simulation is carried out to include quantization, thermal noise effects $\left(\mathrm{F}_{\max }\right.$ is chosen as maximum clock frequency given for $\mathrm{ADC}$ i.e., $\left.250 \mathrm{MHz}\right)$. The following inputs are given for evaluating the dynamic performance of 10-bit TI-SAR ADC. $\mathrm{V}_{\text {in }}=$ $1 \mathrm{~V}_{\mathrm{p}-\mathrm{p}}, \mathrm{F}_{\mathrm{in}}=\mathrm{F}_{\mathrm{s}} / 8(33.6 \mathrm{MHz}), \mathrm{F}_{\mathrm{s}}=250 \mathrm{MS} / \mathrm{s}$ and no. of sample points $(\mathrm{N})$ is 128 . The achieved SFDR is $66 \mathrm{~dB}$ and SNDR of $59.3 \mathrm{~dB}$ (ENOB $=9.6$ bits). This results in a FoM value of 60 fJ/step.

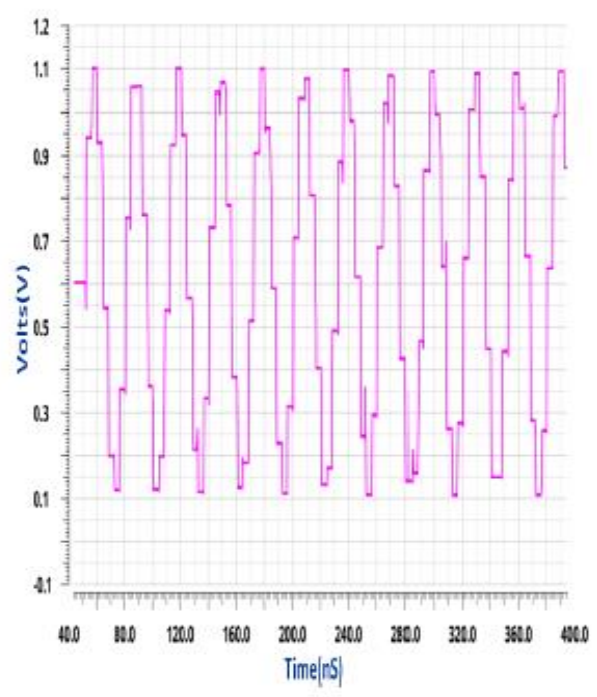

(a)

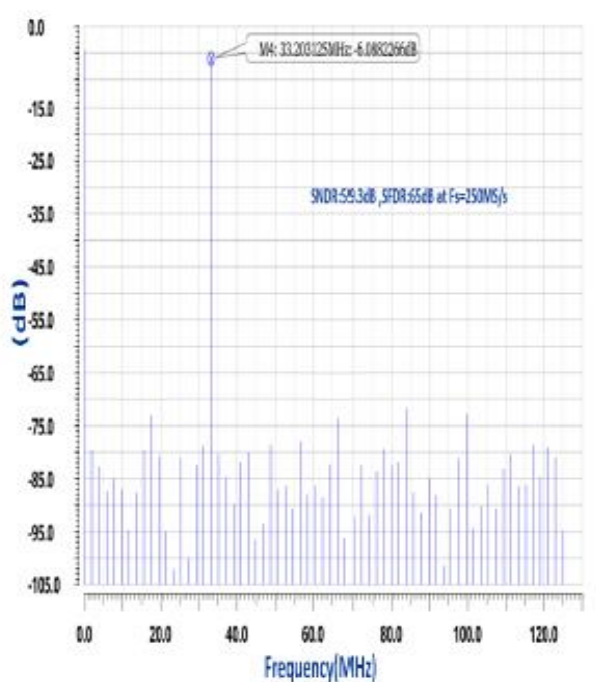

(b)

Figure 19. Single channel TI-SAR ADC (a) Transient response (b) Output Spectrum

\section{Performance Summary ANd Comparison}

Table 1. Performance Summary of 10-bit SAR ADC

\begin{tabular}{|l|l|}
\hline Parameter & Value \\
\hline Process/Technology & UMC $65 \mathrm{~nm}$ CMOS Technology \\
\hline Supply Voltage & $1.2 \mathrm{~V}$ \\
\hline Input Swing (differential) & $2 \mathrm{~V}_{\mathrm{p}-\mathrm{p}}$ \\
\hline Sampling Rate & $15 \mathrm{MS} / \mathrm{s}$ \\
\hline Input Offset Voltage & $\pm 872 \mu \mathrm{V}$ \\
\hline SFDR & $64.5 \mathrm{~dB}$ \\
\hline SNDR & $57 \mathrm{~dB}$ \\
\hline ENOB & $9.2 \mathrm{bits}$ \\
\hline Power consumption & $560 \mu \mathrm{W}$ \\
\hline FoM & $63.5 \mathrm{fJ} /$ Step \\
\hline
\end{tabular}


Table 2. Comparison Table

\begin{tabular}{|l|l|l|l|l|l|}
\hline & This Work & ISSCC [19] & ISSCC [20] & VLSI [21] & $\begin{array}{l}\text { JSSC } \\
{[22]}\end{array}$ \\
\hline Architecture & SAR & SAR & SAR & SAR & SAR \\
\hline Technology $(\mathrm{nm})$ & 65 & 90 & 65 & 90 & 65 \\
\hline Supply Voltage $(\mathrm{V})$ & 1.2 & 1 & 1 & 1 & 1.2 \\
\hline $\begin{array}{l}\text { Sampling Rate } \\
\text { (MS/s) }\end{array}$ & 15 & 50 & 50 & 30 & 50 \\
\hline Resolution (bit) & 10 & 9 & 10 & 10 & 10 \\
\hline Power $(\mu \mathrm{WW})$ & $\mathbf{5 6 0}$ & 700 & 820 & 980 & 826 \\
\hline SFDR $(\mathrm{dB})$ & 64.5 & - & - & 68.16 & 61.8 \\
\hline ENOB (bits) & $\mathbf{9 . 2}$ & 7.8 & 9.16 & 9.16 & 9.18 \\
\hline FoM $(\mathrm{J} / \mathrm{step})$ & $63.5 \mathrm{f}$ & $65 \mathrm{f}$ & $30 \mathrm{f}$ & $57 \mathrm{f}$ & $27 \mathrm{f}$ \\
\hline
\end{tabular}

\section{CONClusion AND Future Work}

This paper describes about various architectures of time-interleaved ADCs, selection of number channels based on sampling rate and sub-ADC architecture. The mathematical model for 14 timeinterleaved $\mathrm{ADC}$ has been developed to analyse the effect of gain, offset and timing mismatch among the channels. The design of 10-bit, $20 \mathrm{MS} / \mathrm{s}$ SAR ADC has been implemented in $65 \mathrm{~nm}$ CMOS Technology. Layout design of 10-bit capacitive DAC, preamplifier based dynamic latch comparator is implemented by using common centroid method and their extracted views are included in simulations. The target sampling rate was $20 \mathrm{MS} / \mathrm{s}$ in this design, however the sampling rate achieved is $15 \mathrm{MS} / \mathrm{s}$. As a result, the 10-bit SAR ADC operate at $15 \mathrm{MS} / \mathrm{s}$ with power consumption of $560 \mu \mathrm{W}$ at $1.2 \mathrm{~V}$ supply and achieves SNDR of $57 \mathrm{~dB}$ (i.e. ENOB 9.2 bits) near Nyquist rate input. The design of 10-bit, 14 time-interleaved (single channel) SAR ADC has been implemented. The achieved SNDR of $59.3 \mathrm{~dB}$ (ENOB $=9.6$ bits) and SFDR of 66 $\mathrm{dB}$ with power consumption of $11.6 \mathrm{~mW}$. This results in a FoM value of $60 \mathrm{fJ} / \mathrm{step}$.

\subsection{Future Work}

The future work following this are -

- Implementation of proposed 4-channel, $1 \mathrm{GS} / \mathrm{s}$ time-interleaved SAR ADC.

- Implementation of calibration techniques to overcome the non-ideal effects in timeinterleaving ADC (offset, gain and timing mismatches among the channels).

\section{ACKNOWLEDGEMENTS}

I would like to express my appreciation and special thanks to my guide Prof. Dinesh K. Sharma, Department of Electrical Engineering, IITB, Mumbai for all of his guidance, support during my work and also to Prof. Ashudeb Dutta, at DARMIC Lab, IIT Hyderabad for giving valuable suggestion for this work.

\section{REFERENCES}

[1] Kenneth C. Dyer, John P. Keane, and Stephen H. Lewis Calibration and Dynamic Matching in Data Converters s Part 2: Time-interleaved analog-to-digital converters and background-calibration challenges, IEEE Solid-state circuits Magazine, Summer 2018. 
[2] Wei Shu, Member, IEEE, and Joseph S. ChangA 1-GS/s 11-Bit SAR-Assisted Pipeline ADC With 59-dB SNDR in 65-nm CMOS, IEEE transactions on circuits and systems-II: Express Briefs, vol. 65, no. 09, Sept.2018.

[3] Jie Fang, Shankar Thirunakkarasu, Member, IEEE, Xuefeng Yu, Fabian Silva-Rivas, Chaoming Zhang, Frank Singor, and Jacob Abraham, Fellow, "A 5-GS/s 10-b 76-mW Time-Interleaved SAR ADC in 28 nm CMOS IEEE J. Solid-State Circuits -I: Regular Papers, vol.64, no.7, July2017

[4] Chithira Ravi, Vineeth Sarma, and Bibhudatta Sahoo, "At Speed Digital Gain Error Calibration of Pipelined ADCs" IEEE transactions on very large scale integration (VLSI) systems, vol. 25, no. 11, november 2017.

[5] Time-interleaved Analog-to-Digital Converters Authors: Louwsma, Simon, van Tuijl, E.D., Nauta, Bram

[6] A $480 \mathrm{~mW} 2.6 \mathrm{GS} / \mathrm{s} 10 \mathrm{~b}$ Time-Interleaved ADC With $48.5 \mathrm{~dB}$ SNDR up to Nyquist in $65 \mathrm{~nm}$ CMOS Kostas Doris, Member, IEEE, Erwin Janssen, Member, IEEE, Claudio Nani, Athon Zanikopoulos, Member, IEEE, and Gerard van der Weide ,IEEE JOURNAL OF SOLID-STATE CIRCUITS, VOL. 46, NO. 12, DECEMBER 2011

[7] A Time-Interleaved Flash-SAR Architecture for High Speed A/D Conversion Ba Ro Saim Sung, Sang-Hyun Cho, Chang-Kyo Lee, Jong-In Kim, and Seung-Tak Ryu School of engineering, Information and Communications University

[8] Design of High-Speed Analog-to-Digital Converters using Low-Accuracy Components Timmy Sundstr" om ,ISBN 978-91-7393-203-5 ,ISSN 0345-7524

[9] Kent, H. L. (2002). Analog to Digital converter testing.

[10] Gustavsson, M. (1998). CMOS A/D converters for telecommunications. LINKOPING STUDIES IN SCIENCE AND TECHNOLOGYDISSERTATIONS-.

[11] Qazi, S. (2010). Study of Time-Interleaved SAR ADC and Implementation of Comparator for High Definition Video ADC in 65nm CMOS Process.

[12] Mehta, N. (2013). Sampling Time Error Calibration for Time-Interleaved ADCs. MASTER OF SCIENCE THESIS, Delft University of Technology.

[13] Liu, C. C., Chang, S. J., Huang, G. Y., and Lin, Y. Z. (2010). A 10-bit 50-MS/s SAR ADC with a monotonic capacitor switching procedure. IEEE Journal of Solid-State Circuits, 45(4), 731-740.

[14] Ginsburg, B. P., and Chandrakasan, A. P. (2007). 500-MS/s 5-bit ADC in 65-nm CMOS with split capacitor array DAC. IEEE Journal of Solid-State Circuits, 42(4), 739-747.

[15] Yue, X. (2013). Determining the reliable minimum unit capacitance for the DAC capacitor array of SAR ADCs. Microelectronics Journal, 44(6), 473-478.

[16] Tsividis, Y. (2002). Mixed analog-digital VLSI devices and technology. World Scientific.

[17] Anderson, T. O. (1972). Optimum control logic for successive approximation analog-to-digital converters. Deep Space Network Progress Report, 13, 168-176.

[18] Rossi, A.,and Fucili, G. (1996). Nonredundant successive approximation register for A/D converters. Electronics letters, 32(12), 1055-1057.

[19] Craninckx, J., \& Van der Plas, G. (2007, February). A 65fJ/conversionstep 0-to-50MS/s 0-to-0.7 mW 9b charge-sharing SAR ADC in 90nm digital CMOS. In Solid-State Circuits Conference, 2007. ISSCC 2007. Digest of Technical Papers. IEEE International (pp. 246-600). IEEE.

[20] M. Yoshioka, K. Ishikawa, T. Takayama, and S. Tsukamoto, "A 10b 50 MS/s 820 mW SAR ADC with on-chip digital calibration," in Proc.IEEE ISSCC Dig. Tech. Papers, Feb. 2010, pp. 384-385.

[21] Huang, G. Y., Chang, S. J., Liu, C. C., \& Lin, Y. Z. (2013). 10-bit 30-MS/s SAR ADC using a switchback switching method. IEEE Transactions on Very Large Scale Integration (VLSI) Systems, 21(3), 584-588.

[22] Liu, C. C., Chang, S. J., Huang, G. Y., \& Lin, Y. Z. (2010). A 10-bit 50-MS/s SAR ADC with a monotonic capacitor switching procedure. IEEE Journal of Solid-State Circuits, 45(4), 731-740.

[23] Gupta, S., Choi, M., Inerfield, M., \& Wang, J. A 1 GS/s 11 b time-interleaved ADC in 0.13muhboxm CMOS. In IEEE ISSCC Dig. Tech. Papers (Vol. 49, pp. 576-577).

[24] Black, W. C., \& Hodges, D. A. (1980). Time interleaved converter arrays. IEEE Journal of Solidstate circuits, 15(6), 1022-1029.

[25] Jamal, S. M., Fu, D., Chang, N. J., Hurst, P. J., \& Lewis, S. H. (2002). A 10-b 120-Msample/s timeinterleaved analog-to-digital converter with digital background calibration. IEEE Journal of SolidState Circuits, 37(12), 1618-1627. 
[26] Ginsburg, B. P., \& Chandrakasan, A. P. (2005, May). An energy-efficient charge recycling approach for a SAR converter with capacitive DAC. In Circuits and Systems, 2005. ISCAS 2005. IEEE International Symposium on (pp. 184-187). IEEE.

[27] Ginsburg, B. P., \& Chandrakasan, A. P. (2007). Dual time-interleaved successive approximation register ADCs for an ultra-wideband receiver. IEEE Journal of Solid-State Circuits, 42(2), 247-257.

[28] Pelgrom, M. J., Tuinhout, H. P., \& Vertregt, M. (1998, December). Transistor matching in analog CMOS applications. In Electron Devices Meeting, 1998. IEDM'98. Technical Digest., International (pp. 915-918). IEEE.

[29] Mulder, J., Ward, C. M., Lin, C. H., Kruse, D., \& Westra, J. R. A 21 mW 8 b 125 MS/s ADC occupying 0.09hboxmm2 in 0.13muhboxm CMOS. In IEEE Int. Solid-State Circuits Conf. Dig. Tech. Papers (pp. 260-261).

[30] Hegong Wei, Member, IEEE, Peng Zhang, Bibhu Datta Sahoo, and Behzad Razavi, Fellow, IEEE “An 8-Bit 4GS/s 120mW CMOS ADC", IEEE J. Solid-State Circuits, vol.49, no.8, Aug.2014.

[31] M. Brandolini et al., "26.6 A 5GS/S 150mW 10b SHA-less pipelined/SAR hybrid ADC in 28nm CMOS,” in ISSCC Dig. Tech. Papers, San Francisco, CA, USA, 2015, pp. 1-3.

\section{AUTHORS}

Shravan Kumar Donthula Currently pursuing $\mathrm{PhD}$ in Micro-Electronics and VLSI Design, IIT Hyderabad with focus on developing Analog and Mixed Signal IC design for low power applications. Prior to this he completed MTech in Micro-electronics and VLSI Design from IITB, Mumbai.

Supravat Debnath Currently pursuing MTech in Integrated Sensor Systems as a Research Assistant under Dr. Ashudeb Dutta, at DARMIC Lab, IIT Hyderabad with focus on developing Analog/RF front-ends for low power, long range communication systems. Previously he completed BTech in Electronics and Comm. engineering from Kalyani Govt. Engg. College.

(C) 2021 By AIRCC Publishing Corporation. This article is published under the Creative Commons Attribution (CC BY) license. 